\title{
IN HALTSVER ZEICHNIS
}

G. Bilkenroth Begrüßungsansprache des Leiters des Internationalen Büros für Gebirgsmechanik bei der Deutschen Akademie der Wissen. schaften zu Berlin . . . . . . . . . . . . . . . . . . . . .

W. HARTKE Begrüßungsansprache des Präsidenten der Deutschen Akademie der Wissenschaften zu Berlin . . . . . . . . . . . . . .

K.-H. Hörer Die Arbeit des Internationalen Büros für Gebirgsmechanik im Jahre 1961 . . . . . . . . . . . . . . . . . . . 11

F. MонR

ST. KNOTHE

Bericht der Arbeitsgruppe „Begriffsbestimmungen“ . . . . . . 13

Bericht der Arbeitsgruppe „Gerätetechnik für Untertagemes. sungen" . . . . . . . . . . . . . . . . 15

R. KVAPIL

Bericht der Arbeitsgruppe „Festigkeitsuntersuchungen“ . . . . 16

Ergebnisse der Diskussionen über die Tätigkeit der Arbeits-

gruppen . . . . . . . . . . . . . . . . . 18

\section{Referate zum Thema}

Kritische Einschätzung gebirgsmechanischer Modellversuche und die Grenzen ihrer praktischen Anwendbarkeit

G. BILKENROTH Zum Rahmenthema ,Kritische Einschätzung gebirgsmechanischer Modellversuche". . . . . . . . . . . . . 25

H. G. Denkhaus Modellversuche in der Gebirgsmechanik . . . . . . . . . . . . 29

R. Kvapil Anwendung der Spannungsoptik bei Forschungsarbeiten auf dem Gebiete der Gebirgsmechanik . . . . . . . . . . . . . 43

F. Martos Einige Bemerkungen über prinzipielle und praktische Probleme gebirgsmechanischer Modellversuche aus äquivalenten Materialien

I. T. MrNčev Ưber den Spannungszustand in einem geschichteten Gebirge um Grubenbaue mit elliptischem, kreisförmigem und trapezförmigem Querschnitt . . . . . . . . . . . . . . 52

J. Коночтек Kritische Einschätzung gebirgsmechanischer Modellversuche bei der Lösung der Stabilität eines Schichtenverbandes der nordböhmischen Tertiärformation . . . . . . . . . . . . . . . . 64

V. SibEK Einige Ergebnisse der Untersuchung von Gebirgsdrücken und Verformungen in weichem Gebirge in situ und an Modellen in der Tschechoslowakischen Sozialistischen Republik und ihre kritische Bewertung . . . . . . . . . . . . . . 72

Zum 3. Ländertreffen des Internationalen Büros für Gebirgsmechanik vorgelegte Arbeiten

Y. HiRAMATSU und $\mathrm{Y}$. OKa

G. OBERTI

L. Gopri
Die Spannungen um einen Schacht oder eine Strecke im Gebirge, das sich im dreidimensionalen Spannungszustand befindet . . . 81 Die Theorie der Modelle unter besonderem Hinweis auf Probleme der Geomechanik . . . . . . . . . . . . . . . . . 101 Anwendung und Auswertung von Untersuchungen an geomechanischen Modellen . . . . . . . . . . . . . . . . . . 106 
S. G. Averšı́ Die Möglichkeiten der Modellierung gebirgsmechanischer Prozesse in der Umgebung bergmännischer Hohlräume . . . . . . . . . 1 13

G. N. KuZnecov Die Modellierung von Gebirgsdruckprozessen mit Hilfe äquivalenter Materialien. . . . . . . . . . . . . . . . . . . . . . . 118

A. J. Surkov Eine Untersuchung der Spannungsverteilung in Schächten in der Nähe von Füllörtern . . . . . . . . . . . . . . . . . . . 123

K. SZÉCH Y Beitrag zur Gebirgsdruckverteilung um einen kreisförmigen Tunnelquerschnitt . . . . . . . . . . . . . . 131

R. RICHTER Fragen spannungsoptischer Untersuchungen in der Gebirgsmechanik . . . . . . . . . . . . . . . . . . . 137

L. Rózsa und Bemessung von zylindrischen Stollenausbetonierungen mit beson-

K. Kovícs derem Bezug auf die Stützwirkung des umgebenden Bodens. . . . 142

Wissenschaftliche Aussprache

Diskussion zum Rahmenthema Kritische Einschätzung gebirgsmechanischer Modell-

versuche und die Grenzen ihrer praktischen Anwendbarkeit . . . . . . . . . . . . 153

Arbeitsprogramm - Schlußwort

Arbeitsprogramm des Internationalen Büros für Gebirgsmechanik für das Jahr 1962193

Exkursion in den Mansfelder Kupferschieferbergbau

W. Dietze Neuere Ergebnisse der Gebirgsdruckforschung im Mansfelder Kupferschieferbergbau . . . . . . . . . . . . . . . . . . 199

Teilnehmerverzeichnis ... . . . . . . . . . . . . . . 205 